\title{
On extension of classical Baer results to Poisson algebras*
}

\section{A. Kurdachenko, A. A. Pypka, and I. Ya. Subbotin}

Dedicated to the 60th anniversary of the Department of Algebra and Mathematical Logic of Taras Shevchenko National University of Kyiv

ABSTRACT. In this paper we prove that if $P$ is a Poisson algebra and the $n$-th hypercenter (center) of $P$ has a finite codimension, then $P$ includes a finite-dimensional ideal $K$ such that $P / K$ is nilpotent (abelian). As a corollary, we show that if the $n$-th hypercenter of a Poisson algebra $P$ (over some specific field) has a finite codimension and $P$ does not contain zero divisors, then $P$ is an abelian algebra.

\section{Introduction}

Let $P$ be a vector space over a field $F$. Then $P$ is called a Poisson algebra, if $P$ has two additional binary operations: the multiplication and $[$,$] such that the following conditions hold:$

$$
\begin{gathered}
a b=b a, \quad(a b) c=a(b c), \quad a(b+c)=a b+a c, \quad(\lambda a) b=\lambda(a b)=a(\lambda b) ; \\
{[a+b, c]=[a, c]+[b, c], \quad[a, b+c]=[a, b]+[a, c],} \\
{[\lambda a, b]=\lambda[a, b]=[a, \lambda b], \quad[a, a]=0,} \\
{[[a, b], c]+[[b, c], a]+[[c, a], b]=0 ;} \\
{[a b, c]=a[b, c]+b[a, c]}
\end{gathered}
$$

for all elements $a, b, c \in P, \lambda \in F$.

*The first two authors are supported by the National Research Foundation of Ukraine (grant no. 2020.02/0066).

2020 MSC: 17B63,17B65.

Key words and phrases: Poisson algebra, Lie algebra, subalgebra, ideal, center, hypercenter, zero divisor, finite dimension, nilpotency. 
If we will consider $P$ as an associative and commutative algebra by the outer multiplication, addition and multiplication, then we will denote it by $P(+, \cdot)$. If we will consider $P$ as a Lie algebra by outer multiplication, addition and Lie brackets, then we will denote it by $P(+,[]$,$) .$

Poisson algebras arose from the study of Poisson geometry [27,52]. It has appeared in an extremely wide range of areas in mathematics and physics, such as classical and quantum mechanics [1, 12, 36], quantum groups [11, 13], quantization theory [4, 5, 20,21], Poisson manifolds [26, 48], algebraic geometry $[6,18,38]$, operads $[16,19,32]$. Some of the first works where concrete Poisson algebras appeared were [7, 9, 39, 40,51], while one of the first works, where the study of the properties of abstract Poisson algebras began, was the work [2]. Poisson algebras have been and are being studied very intensively by many authors and from various points of view (see, for example, the articles [10, 14, 15, 17, 19, 28-31,33, 34,37, 4147,49] and the book [8]). This current paper is dedicated to extending to Poisson algebras some results that became already classical in different algebraic structures. The issue that will be discussed here has its sources in articles [3,35]. They showed that if the center of a group has a finite index, then its derived subgroup is finite. This result became the starting point for an interesting and broad topic, involving not only groups, but also other algebraic structures, among which were non-associative algebras (Lie algebras and Leibniz algebras) (see a survey [25]). In particular, in the paper [50] has been proved that if the center of a Lie algebra has finite codimension, then its derived ideal has finite dimension. A situation with the center and derived subalgebra in Poisson algebras has significant differences, in the Poisson algebras the center and derived subalgebra are not ideals. Nevertheless, for Poisson algebras we obtained a similar result.

Let $P$ be a Poisson algebra over a field $F$. As usual, a subset $B$ of $P$ is called a subalgebra of $P$ if $B$ is a subspace of $P$ and $x y,[x, y] \in B$ for every elements $x, y \in B$.

A subset $L$ of $P$ is called an ideal of $P$ if $L$ is a subspace of $P$ and $x b,[x, b] \in L$ for every elements $b \in L$ and $x \in P$.

A Poisson algebra $P$ is called simple if it has only two ideals $\langle 0\rangle$ and $P$. The Poisson algebra $P$ is called abelian, if $[x, y]=0$ for all $x, y \in P$.

Let $P$ be a Poisson algebra. Define the lower central series of $P$

$$
P=\gamma_{1}(P) \geqslant \gamma_{2}(P) \geqslant \ldots \gamma_{\alpha}(P) \geqslant \gamma_{\alpha+1}(P) \geqslant \ldots \gamma_{\delta}(P)
$$

by the following rule: $\gamma_{1}(P)=P, \gamma_{2}(P)=[P, P]$, recursively $\gamma_{\alpha+1}(P)=$ $\left[\gamma_{\alpha}(P), P\right]$ for all ordinals $\alpha$, and $\gamma_{\lambda}(P)=\bigcap_{\mu<\lambda} \gamma_{\mu}(P)$ for limit ordinals 
$\lambda$. The last term $\gamma_{\delta}(P)$ is called the lower hypocenter of $P$. We have $\gamma_{\delta}(P)=\left[\gamma_{\delta}(P), P\right]$.

As usually, we say that a Poisson algebra $P$ is nilpotent, if there exists a positive integer $k$ such that $\gamma_{k}(P)=\langle 0\rangle$. More precisely, $P$ is said to be nilpotent of nilpotency class $c$ if $\gamma_{c+1}(P)=\langle 0\rangle$, but $\gamma_{c}(P) \neq\langle 0\rangle$. We denote the nilpotency class of $P$ by $\operatorname{ncl}(P)$.

Put

$$
\zeta(P)=\{z \in P \mid[z, x]=0 \text { for every element } x \in P\} .
$$

The subset $\zeta(P)$ is called the center of $P$.

Starting from the center we can construct the upper central series

$$
\langle 0\rangle=\zeta_{0}(P) \leqslant \zeta_{1}(P) \leqslant \ldots \zeta_{\alpha}(P) \leqslant \zeta_{\alpha+1}(P) \leqslant \ldots \zeta_{\gamma}(P)=\zeta_{\infty}(P)
$$

of a Poisson algebra $P$ by the following rule: $\zeta_{1}(P)=\zeta(P)$ is the center of $P$, recursively $\zeta_{\alpha+1}(P) / \zeta_{\alpha}(P)=\zeta\left(P / \zeta_{\alpha}(P)\right)$ for all ordinals $\alpha$, and $\zeta_{\lambda}(P)=\bigcup_{\mu<\lambda} \zeta_{\mu}(P)$ for limit ordinals $\lambda$. We remark that each term of this series is a subalgebra of $P$, which is an ideal of a Lie algebra $P(+,[]$,$) .$ The last term $\zeta_{\infty}(P)$ of this series is called the upper hypercenter of $P$.

It is a well-known that in nilpotent Lie algebras the lower and the upper central series have the same length.

For Poisson algebras we obtained the following result.

Theorem A. Let $P$ be a Poisson algebra over a field $F$. Suppose that the center of $P$ has a finite codimension $d$. Then $P$ includes an ideal $K$ of finite dimension at most $\frac{1}{2} d\left(d^{2}-1\right)$ such that $P / K$ is abelian.

In the paper [3] it was proved that if the hypercenter $\zeta_{n}(G)$ of a group $G$ has a finite index, then its $(n+1)$ th hypocenter $\gamma_{n+1}(G)$ is finite. This result was also extended to other algebraic structures, in particular, on Lie algebras [24] and Leibniz algebras [22,23]. For the Poisson algebras we obtain the following result.

Theorem B. Let $P$ be a Poisson algebra over a field $F$. Suppose that hypercenter $\zeta_{n}(P)$ has a finite codimension $d$. Then $P$ includes an ideal $K$, having finite dimension at most $d^{n+1}(1+d)$, such that $P / K$ is nilpotent of nilpotency class at most n.

Here are some interesting corollaries.

Corollary B1. Let $P$ be a Poisson algebra over a field $F$. Suppose that $\operatorname{char}(F)=p$ is a prime and $F^{p}=F$. If hypercenter $\zeta_{n}(P)$ has finite codimension and $P$ does not contain zero divisors, then $P$ is abelian.

Corollary B2. Let $P$ be a Poisson algebra over a finite field $F$. If hypercenter $\zeta_{n}(P)$ has finite codimension and $P$ does not contain zero divisors, then $P$ is abelian. 
Corollary B3. Let $P$ be a Poisson algebra over a locally finite field $F$. If hypercenter $\zeta_{n}(P)$ has finite codimension and $P$ does not contain zero divisors, then $P$ is abelian.

\section{Some preliminary results}

Let $P$ be a Poisson algebra. The equality $[a, a]=0$ implies that

$$
[a, b]=-[b, a]
$$

Then

$$
[c, a b]=-[a b, c]=-a[b, c]-b[a, c]=a[c, b]+b[c, a] .
$$

We now point out several consequences of these basic equalities. We have

$$
[a b c, d]=a[b c, d]+b c[a, d]=a b[c, d]+a c[b, d]+b c[a, d] .
$$

Using ordinary induction, we obtain

$$
\begin{gathered}
{\left[a_{1} a_{2} a_{3} a_{4} \ldots a_{n}, x\right]=a_{2} a_{3} a_{4} \ldots a_{n}\left[a_{1}, x\right]+a_{1} a_{3} a_{4} \ldots a_{n}\left[a_{2}, x\right]} \\
+a_{1} a_{2} a_{4} \ldots a_{n}\left[a_{3}, x\right]+\ldots+a_{1} a_{2} a_{3} \ldots a_{n-1}\left[a_{n}, x\right]
\end{gathered}
$$

Furthermore

$$
\begin{aligned}
{[x y, a b] } & =x[y, a b]+y[x, a b]=x(a[y, b]+b[y, a])+y(a[x, b]+b[x, a]) \\
& =x a[y, b]+x b[y, a]+y a[x, b]+y b[x, a] .
\end{aligned}
$$

We have also

$$
\begin{gathered}
{\left[c^{2}, d\right]=[c c, d]=c[c, d]+c[c, d]=2 c[c, d]} \\
{\left[c^{3}, d\right]=\left[c^{2} c, d\right]=c^{2}[c, d]+c\left[c^{2}, d\right]=c^{2}[c, d]+c(2 c[c, d])=3 c^{2}[c, d] .}
\end{gathered}
$$

Suppose that we have already proved that $\left[c^{n}, d\right]=n c^{n-1}[c, d]$. Then

$$
\begin{aligned}
{\left[c^{n+1}, d\right] } & =\left[c^{n} c, d\right]=c^{n}[c, d]+c\left[c^{n}, d\right]=c^{n}[c, d]+c\left(n c^{n-1}[c, d]\right) \\
& =(n+1) c^{n}[c, d] .
\end{aligned}
$$

Thus for every positive integer $k$ we proved that $\left[c^{k}, d\right]=k c^{k-1}[c, d]$. Further

$$
\begin{gathered}
{\left[c^{k}, d^{2}\right]=\left[c^{k}, d d\right]=d\left[c^{k}, d\right]+d\left[c^{k}, d\right]=2 d\left[c^{k}, d\right]=2 k d c^{k-1}[c, d],} \\
{\left[c^{k}, d^{3}\right]=\left[c^{k}, d d^{2}\right]=d\left[c^{k}, d^{2}\right]+d^{2}\left[c^{k}, d\right]} \\
=2 k d^{2} c^{k-1}[c, d]+k d^{2} c^{k-1}[c, d]=3 k d^{2} c^{k-1}[c, d]
\end{gathered}
$$


Suppose that we have already proved that $\left[c^{k}, d^{t}\right]=k t d^{t-1} c^{k-1}[c, d]$. Then

$$
\begin{aligned}
& {\left[c^{k}, d^{t+1}\right]=\left[c^{k}, d d^{t}\right]} \\
& \quad=d\left[c^{k}, d^{t}\right]+d^{t}\left[c^{k}, d\right]=d\left(k t d^{t-1} c^{k-1}[c, d]\right)+d^{t}\left(k c^{k-1}[c, d]\right) \\
& \quad=k t d^{t} c^{k-1}[c, d]+k d^{t} c^{k-1}[c, d]=k(t+1) d^{t} c^{k-1}[c, d] .
\end{aligned}
$$

Thus for every positive integers $k$ and $s$ we proved the equality $\left[c^{k}, d^{s}\right]=$ $k s d^{s-1} c^{k-1}[c, d]$.

Let $A$ and $U$ be Poisson algebras over a field $F$. Then a mapping $f: A \rightarrow U$ is called a homomorphism, if

$$
\begin{aligned}
& f(\lambda a)=\lambda f(a), f(a+b)=f(a)+f(b), \\
& f(a b)=f(a) f(b), f([a, b])=[f(a), f(b)]
\end{aligned}
$$

for all elements $a, b \in A, \lambda \in F$.

As usual, an injective homomorphism is called a monomorphism, a surjective homomorphism is called an epimorphism and bijective homomorphism is called an isomorphism.

Proposition 1. Let $A$ be an associative and commutative algebra over a field $F$, generating by a subset $S$. Suppose that on $A$ is defined a bilinear operation $[$,$] satisfying conditions [a, a]=0$ and $[a b, c]=a[b, c]+b[a, c]$. Then $A$ is a Poisson algebra if and only if $[[a, b], c]+[[b, c], a]+[[c, a], b]=0$ for all elements $a, b, c \in S$.

Proof. If $A$ is a Poisson algebra, then $[[a, b], c]+[[b, c], a]+[[c, a], b]=0$ for arbitrary elements $a, b, c$, in particular, this equality is valid for elements $a, b, c \in S$.

Conversely, suppose that $[[a, b], c]+[[b, c], a]+[[c, a], b]=0$ for all elements $a, b, c \in S$. Let $d \in S$. We have

$$
\begin{aligned}
& {[[a, b], c d]+[[b, c d], a]+[[c d, a], b] } \\
&= c[[a, b], d]+d[[a, b], c]+[c[b, d]+d[b, c], a]+[c[d, a]+d[c, a], b] \\
&= c[[a, b], d]+d[[a, b], c]+[c[b, d], a] \\
&+[d[b, c], a]+[c[d, a], b]+[d[c, a], b] \\
&= c[[a, b], d]+d[[a, b], c]+c[[b, d], a]+[b, d][c, a]+d[[b, c], a] \\
&+[b, c][d, a]+c[[d, a], b]+[d, a][c, b]+d[[c, a], b]+[c, a][d, b] \\
&= c([[a, b], d]+[[b, d], a]+[[d, a], b])+d([[a, b], c]+[[b, c], a] \\
&+[[c, a], b])+[b, d][c, a]+[b, c][d, a]+[d, a][c, b]+[c, a][d, b]=0 .
\end{aligned}
$$


Using an ordinary induction, we obtain that

$$
\left[[a, b], c_{1} \ldots c_{n}\right]+\left[\left[b, c_{1} \ldots c_{n}\right], a\right]+\left[\left[c_{1} \ldots c_{n}, a\right], b\right]=0
$$

for all elements $a, b, c_{1}, \ldots, c_{n} \in S$. Further

$$
\begin{aligned}
& {\left[\left[a_{1} a_{2}, b\right], c\right]+\left[[b, c], a_{1} a_{2}\right]+\left[\left[c, a_{1} a_{2}\right], b\right]} \\
& =\left[a_{1}\left[a_{2}, b\right]+a_{2}\left[a_{1}, b\right], c\right]+a_{1}\left[[b, c], a_{2}\right]+a_{2}\left[[b, c], a_{1}\right] \\
& \quad+\left[a_{1}\left[c, a_{2}\right]+a_{2}\left[c, a_{1}\right], b\right] \\
& =\left[a_{1}\left[a_{2}, b\right], c\right]+\left[a_{2}\left[a_{1}, b\right], c\right]+a_{1}\left[[b, c], a_{2}\right] \\
& \quad+a_{2}\left[[b, c], a_{1}\right]+\left[a_{1}\left[c, a_{2}\right], b\right]+\left[a_{2}\left[c, a_{1}\right], b\right] \\
& =a_{1}\left[\left[a_{2}, b\right], c\right]+\left[a_{2}, b\right]\left[a_{1}, c\right]+a_{2}\left[\left[a_{1}, b\right], c\right]+\left[a_{1}, b\right]\left[a_{2}, c\right] \\
& \quad+a_{1}\left[[b, c], a_{2}\right]+a_{2}\left[[b, c], a_{1}\right]+a_{1}\left[\left[c, a_{2}\right], b\right] \\
& \quad+\left[c, a_{2}\right]\left[a_{1}, b\right]+a_{2}\left[\left[c, a_{1}\right], b\right]+\left[c, a_{1}\right]\left[a_{2}, b\right] \\
& =a_{1}\left(\left[\left[a_{2}, b\right], c\right]+\left[[b, c], a_{2}\right]+\left[\left[c, a_{2}\right], b\right]\right)+ \\
& \quad+a_{2}\left(\left[\left[a_{1}, b\right], c\right]+\left[[b, c], a_{1}\right]+\left[\left[c, a_{1}\right], b\right]\right)+ \\
& \quad+\left[a_{2}, b\right]\left[a_{1}, c\right]+\left[a_{1}, b\right]\left[a_{2}, c\right]+\left[c, a_{2}\right]\left[a_{1}, b\right]+\left[c, a_{1}\right]\left[a_{2}, b\right]=0 .
\end{aligned}
$$

Using an ordinary induction, we obtain that

$$
\left[\left[a_{1} \ldots a_{k}, b\right], c\right]+\left[[b, c], a_{1} \ldots a_{k}\right]+\left[\left[c, a_{1} \ldots a_{k}\right], b\right]=0
$$

for all elements $a_{1}, \ldots, a_{k}, b, c \in S$. Taking into account what was proved above, we obtain

$$
\left[\left[a_{1} \ldots a_{k}, b\right], c_{1} \ldots c_{n}\right]+\left[\left[b, c_{1} \ldots c_{n}\right], a_{1} \ldots a_{k}\right]\left[\left[c_{1} \ldots c_{n}, a_{1} \ldots a_{k}\right], b\right]=0
$$

for all elements $a_{1}, \ldots, a_{k}, b, c_{1}, \ldots, c_{n} \in S$. Using similar reasoning, we prove the equality

$$
\left[\left[a, b_{1} \ldots b_{t}\right], c\right]+\left[\left[b_{1} \ldots b_{t}, c\right], a\right]+\left[[c, a], b_{1} \ldots b_{t}\right]=0
$$

for all elements $a, b_{1}, \ldots, b_{t}, c \in S$.

From this we already get

$$
\begin{aligned}
& {\left[\left[a_{1} \ldots a_{k}, b_{1} \ldots b_{t}\right], c_{1} \ldots c_{n}\right]+\left[\left[b_{1} \ldots b_{t}, c_{1} \ldots c_{n}\right], a_{1} \ldots a_{k}\right]} \\
& +\left[\left[c_{1} \ldots c_{n}, a_{1} \ldots a_{k}\right], b_{1} \ldots b_{t}\right]=0 .
\end{aligned}
$$

for all elements $a_{1}, \ldots, a_{k}, b_{1}, \ldots, b_{t}, c_{1}, \ldots, c_{n} \in S$.

Every element $x$ of $A$ has a form $x=y_{1}+\ldots+y_{m}$ where $y_{j}=$ $\alpha v_{1, j} \ldots v_{r, j}$ where $\alpha \in F, v_{1, j}, \ldots, v_{r, j} \in S$. Since an operation [ , ] is 
bilinear, from proved above it follows that Jacobi identity is valid for all elements of $A$.

Proposition 2. Let $A$ be an arbitrary Poisson algebra over a field $F$. Then there exists a Poisson algebra $S$ over a field $F$ having a multiplicative identity element and monomorphism $f: A \rightarrow S$. Moreover, $\operatorname{Im}(f)$ is an ideal of $S$.

Proof. If $A$ has an identity element by multiplication, then all is proved. Therefore we will suppose that $A$ has no an identity element. Put $S=A \times F$ and define on $S$ the following operations. Put

$$
\begin{aligned}
\gamma(a, \lambda) & =(\gamma a, \gamma \lambda),(a, \lambda)+(b, \mu)=(a+b, \lambda+\mu), \\
(a, \lambda)(b, \mu) & =(a b+\lambda b+\mu a, \lambda \mu),[(a, \lambda),(b, \mu)]=([a, b], 0)
\end{aligned}
$$

for all elements $a, b \in A, \lambda, \mu, \gamma \in F$.

As in Algebra Theory it is possible to prove that by the outer multiplication, addition and multiplication $S$ is an associative and commutative algebra and the pair $\left(0,1_{F}\right)$ is its identity element. Furthermore,

$$
\begin{gathered}
{[(a, \lambda),(a, \lambda)]=([a, a], 0)=(0,0),} \\
{[[(a, \lambda),(b, \mu)],(c, \gamma)]+[[(b, \mu),(c, \gamma)],(a, \lambda)]+[[(c, \gamma),(a, \lambda)],(b, \mu)]} \\
=[([a, b], 0),(c, \gamma)]+[([b, c], 0),(a, \lambda)]+[([c, a], 0),(b, \mu)] \\
=([[a, b], c], 0)+([[b, c], a], 0)+([[c, a], b], 0) \\
=([[a, b], c]+[[b, c], a]+[[c, a], b], 0)=(0,0), \\
{[(a, \lambda)(b, \mu),(c, \gamma)]=[(a b+\lambda b+\mu a, \lambda \mu),(c, \gamma)]=([a b+\lambda b+\mu a, c], 0)} \\
=([a b, c]+[\lambda b, c]+[\mu a, c], 0)=([a b, c]+\lambda[b, c]+\mu[a, c], 0), \\
(a, \lambda)[(b, \mu),(c, \gamma)]+(b, \mu)[(a, \lambda),(c, \gamma)] \\
=(a, \lambda)([b, c], 0)+(b, \mu)([a, c], 0) \\
=(a[b, c]+\lambda[b, c], 0)+(b[a, c]+\mu[a, c], 0) \\
=(a[b, c]+\lambda[b, c]+b[a, c]+\mu[a, c], 0) .
\end{gathered}
$$

Since $[a b, c]=a[b, c]+b[a, c]$, we obtain

$$
[(a, \lambda)(b, \mu),(c, \gamma)]=(a, \lambda)[(b, \mu),(c, \gamma)]+(b, \mu)[(a, \lambda),(c, \gamma)] .
$$

It follows that by the above defined operations, $S$ is a Poisson algebra.

Consider now the mapping $f: A \rightarrow S$, defined by the rule $f(a)=(a, 0)$ for all elements $a \in A$. We have

$$
f(a+b)=(a+b, 0)=(a, 0)+(b, 0)=f(a)+f(b),
$$




$$
\begin{gathered}
f(\lambda a)=(\lambda a, 0)=\lambda(a, 0)=\lambda f(a), \\
f(a b)=(a b, 0)=(a, 0)(b, 0)=f(a) f(b), \\
f([a, b])=([a, b], 0)=[(a, 0),(b, 0)]=[f(a), f(b)] .
\end{gathered}
$$

These equalities show that $f$ is a homomorphism. Clearly $f$ is injective, so that $f$ is a monomorphism. Hence $f(A)$ is a subalgebra of $S$ and $A$ is isomorphic to $S$.

Finally, let $(x, \alpha)$ be an arbitrary element of $S, a$ be an arbitrary element of $A$, then

$$
\begin{gathered}
(x, \alpha)(a, 0)=(x a+\alpha a, 0) \in f(A), \\
{[(x, \alpha),(a, 0)]=([x, a], 0) \in f(A) .}
\end{gathered}
$$

Thus $\operatorname{Im}(f)$ is an ideal of $S$.

This Proposition allows us to consider farther only Poisson algebras having the multiplicative identity element $1_{P}$.

If $B, C$ are the subspaces of a Poisson algebra $P$, then let

- $B+C=\{b+c \mid$ for all elements $b \in B, c \in C\}$,

- $B C$ be the subspace of $P$ generated by the subset

$$
\{b c \mid \text { for all elements } b \in B, c \in C\} \text {, }
$$

- $[B, C]$ be the subspace of $P$ generated by the subset

$$
\{[b, c] \mid \text { for all elements } b \in B, c \in C\} \text {. }
$$

Clearly, $B C$ is a subset of $P$ consisting of elements of the type

$$
a_{1} b_{1}+\ldots+a_{n} b_{n}
$$

where $a_{1}, \ldots, a_{n} \in B, b_{1}, \ldots, b_{n} \in C$.

Similarly, $[B, C]$ is a subset of $P$ consisting of elements of the type

$$
\left[a_{1}, b_{1}\right]+\ldots+\left[a_{n}, b_{n}\right]
$$

where $a_{1}, \ldots, a_{n} \in B, b_{1}, \ldots, b_{n} \in C$.

Proposition 3. Let $P$ be a Poisson algebra over a field $F$.

(i) If $B$ is a subalgebra of $P$ and $C$ is an ideal of $P$, then $B+C, B C$ are subalgebras of $P$. Moreover, if $B, C$ are ideals of $P$, then $B+C$, $B C$ are ideals of $P$.

(ii) The center $\zeta(P)$ is a subalgebra of $P$, which is an ideal of Lie algebra $P(+,[]$,$) .$ 
(iii) The center $\zeta(P)$ contains every idempotent of $P$, in particular, $1_{P} \in \zeta(P)$.

(iv) If $P$ is not a simple algebra, then $P$ has a proper non-zero maximal ideal.

(v) For every element $x \in P$ a subset $x P=\{x y \mid y \in P\}$ is a subalgebra of $P$ and an ideal of $P(+, \cdot)$.

Proof. (i) Let $x, y \in B+C$, then $x=b+c, y=u+v$ where $b, u \in B$, $c, v \in C$. Then

$$
\begin{gathered}
\lambda x=\lambda(b+c)=\lambda b+\lambda c \in B+C \\
x-y=(b+c)-(u+v)=(b-u)+(c-v) \in B+C, \\
x y=(b+c)(u+v)=b u+(c u+b v+c v) \in B+C, \\
{[x, y]=[b+c, u+v]=[b, u]+([c, u]+[b, v]+[c, v]) \in B+C .}
\end{gathered}
$$

These equalities show that $B+C$ is a subalgebra of $P$.

Every element $x$ of $B C$ has a form $x=b_{1} c_{1}+\ldots+b_{n} c_{n}$ where $b_{1}, \ldots, b_{n} \in B, c_{1}, \ldots, c_{n} \in C$. If $\lambda \in F$, then

$$
\lambda x=\lambda\left(b_{1} c_{1}+\ldots+b_{n} c_{n}\right)=\left(\lambda b_{1}\right) c_{1}+\ldots+\left(\lambda b_{n}\right) c_{n} \in B C,
$$

If $y$ is another element of $B C$, then $y=a_{1} d_{1}+\ldots+a_{k} d_{k}, a_{1}, \ldots, a_{k} \in B$, $d_{1}, \ldots, d_{k} \in C$. Then

$$
\begin{gathered}
x-y=\left(b_{1} c_{1}+\ldots+b_{n} c_{n}\right)-\left(a_{1} d_{1}+\ldots+a_{k} d_{k}\right) \in B C, \\
x y=\left(\sum_{1 \leqslant j \leqslant n} b_{j} c_{j}\right)\left(\sum_{1 \leqslant m \leqslant k} a_{m} d_{m}\right)=\sum_{\substack{1 \leqslant j \leqslant n \\
1 \leqslant m \leqslant k}}\left(b_{j} a_{m}\right)\left(c_{j} d_{m}\right) \in B C, \\
{[x, y]=\left[\sum_{1 \leqslant j \leqslant n} b_{j} c_{j}, \sum_{1 \leqslant m \leqslant k} a_{m} d_{m}\right]=\sum_{\substack{1 \leqslant j \leqslant n \\
1 \leqslant m \leqslant k}}\left[b_{j} c_{j}, a_{m} d_{m}\right] .}
\end{gathered}
$$

We have

$$
\left[b_{j} c_{j}, a_{m} d_{m}\right]=b_{j} a_{m}\left[c_{j}, d_{m}\right]+b_{j} d_{m}\left[c_{j}, a_{m}\right]+c_{j} a_{m}\left[b_{j}, d_{m}\right]+c_{j} d_{m}\left[b_{j}, a_{m}\right] .
$$

Since $C$ is an ideal, $\left[c_{j}, d_{m}\right],\left[c_{j}, a_{m}\right],\left[b_{j}, d_{m}\right], d_{m}\left[c_{j}, a_{m}\right], c_{j}\left[b_{j}, d_{m}\right]$, $d_{m} c_{j} \in C$, so that $b_{j} a_{m}\left[c_{j}, d_{m}\right], b_{j} d_{m}\left[c_{j}, a_{m}\right], c_{j} a_{m}\left[b_{j}, d_{m}\right] \in B C$. Since $B$ is a subalgebra, $\left[b_{j}, a_{m}\right] \in B$, so that $c_{j} d_{m}\left[b_{j}, a_{m}\right] \in B C$. Thus we obtain that $\left[b_{j} c_{j}, a_{m} d_{m}\right] \in B C$. It is true for all $j, m, 1 \leqslant j \leqslant n, 1 \leqslant m \leqslant k$, so that $[x, y] \in B C$. These inclusions show that $B C$ is a subalgebra of $P$. 
Suppose now that $B, C$ are ideals of $P$. By above proved $B+C$ and $B C$ are subalgebras of $P$. Let $x \in P, y \in B+C$, then $y=b+c$ where $b \in B, c \in C$. Then

$$
\begin{gathered}
x y=x(b+c)=x b+x c \in B+C, \\
{[x, y]=[x, b+c]=[x, b]+[x, c] \in B+C .}
\end{gathered}
$$

These equalities show that $B+C$ is an ideal of $P$.

Let $x \in P, y \in B C$, then $y=b_{1} c_{1}+\ldots+b_{n} c_{n}$ where $b_{1}, \ldots, b_{n} \in B$, $c_{1}, \ldots, c_{n} \in C$. Then

$$
\begin{gathered}
x y=x\left(\sum_{1 \leqslant j \leqslant n} b_{j} c_{j}\right)=\sum_{1 \leqslant j \leqslant n}\left(x b_{j}\right) c_{j} \in B C, \\
{[x, y]=\left[x, \sum_{1 \leqslant j \leqslant n} b_{j} c_{j}\right]=\sum_{1 \leqslant j \leqslant n}\left[x, b_{j} c_{j}\right]} \\
=\sum_{1 \leqslant j \leqslant n}\left(b_{j}\left[x, c_{j}\right]+c_{j}\left[x, b_{j}\right]\right) \in B C .
\end{gathered}
$$

These equalities show that $B C$ is an ideal of $P$.

(ii) Let $x$ be an arbitrary element of $P$ and $z \in \zeta(P)$. If $\lambda \in F$, then

$$
[x, \lambda z]=\lambda[x, z]=0,
$$

so that $\lambda z \in \zeta(P)$.

If $y$ is another element of $\zeta(P)$, then

$$
[x, z-y]=[x, z]-[x, y]=0,
$$

so that $z-y \in \zeta(P)$. Further

$$
[x, z y]=z[x, y]+y[z, x]=0,
$$

so that $z y \in \zeta(P)$. Furthermore

$$
[[z, y], x]+[[y, x], z]+[[x, z], y]=0 .
$$

Since $[y, x]=0=[x, z]$, we obtain that $[[z, y], x]=0$, so that $[z, y] \in \zeta(P)$. The last inclusion shows that $\zeta(P)$ is a subalgebra of $P$.

(iii) Let $e$ be an idempotent of $P$. For an arbitrary element $x$ of $P$ we obtain

$$
[e, x]=[e e, x]=e[e, x]+e[e, x]=2 e[e, x] .
$$


If $2 e[e, x]=0$, all is proved. Suppose that $2 e[e, x] \neq 0$. We have $[e, x]-$ $2 e[e, x]=0$. It follows that

$0=2 e([e, x]-2 e[e, x])=2 e[e, x]-4 e^{2}[e, x]=2 e[e, x]-4 e[e, x]=-2 e[e, x]$.

Thus $2 e[e, x]=0$.

(iv) Indeed, if $P$ is not simple, then $P$ includes a proper non-zero ideal $K$. Let

$$
\mathfrak{I}=\{Y \mid Y \text { is a proper non-zero ideal of } P\} .
$$

Family $\mathfrak{I}$ is not empty. Since $1_{P} \notin Y$ for each $Y \in \mathfrak{I}$, an union of every linear ordered (by inclusion) subset of $\mathfrak{I}$ belong to $\mathfrak{I}$. By Zorn Lemma a family $\mathfrak{I}$ has a maximal element.

(v) Clearly, $x P$ is an ideal of associative algebra $P(+, \cdot)$. Let $y, z \in x P$, then $y=x u, z=x v$ for some elements $u, v \in P$. We have

$$
\begin{aligned}
{[y, z] } & =[x u, x v]=x[u, x v]+u[x, x v] \\
& =x[u, x v]+u x[x, v]+u v[x, x]=x([u, x v]+u[x, v]) .
\end{aligned}
$$

Thus $[x P, x P] \leqslant x P$, so that $x P$ is a subalgebra of $P$.

Consider some examples of Poisson algebras.

Example 1. If $L$ is a Lie algebra, then put $x y=0$ for all $x, y \in L$. Then, clearly, $L$ is a Poisson algebra.

On the other hand, let $A$ be an associative and commutative algebra. Put $[x, y]=0$. Then, clearly, $L$ is a Poisson algebra. These algebras are called trivial.

Example 2. Let $F$ be a field and $P=F[x, y]$ be a polynomial algebra with two variables $x, y$. Let $f(x, y)$ be an arbitrary polynomial. We denote by $f_{x}^{\prime}$ (respectively, $f_{y}^{\prime}$ ) the derivative of the polynomial $f(x, y)$ with respect to variable $x$ (respectively, $y$ ). Put now

$$
[f, g]=f_{x}^{\prime} g_{y}^{\prime}-f_{y}^{\prime} g_{x}^{\prime}
$$

We have

$$
[\lambda f, g]=(\lambda f)_{x}^{\prime} g_{y}^{\prime}-(\lambda f)_{y}^{\prime} g_{x}^{\prime}=\lambda f_{x}^{\prime} g_{y}^{\prime}-\lambda f_{y}^{\prime} g_{x}^{\prime}=\lambda\left(f_{x}^{\prime} g_{y}^{\prime}-f_{y}^{\prime} g_{x}^{\prime}\right)=\lambda[f, g]
$$

for every element $\lambda \in F$. Similarly, $[f, \lambda g]=\lambda[f, g]$.

$$
\begin{aligned}
{[f+h, g] } & =(f+h)_{x}^{\prime} g_{y}^{\prime}-(f+h)_{y}^{\prime} g_{x}^{\prime}=f_{x}^{\prime} g_{y}^{\prime}+h_{x}^{\prime} g_{y}^{\prime}-f_{y}^{\prime} g_{x}^{\prime}-h_{y}^{\prime} g_{x}^{\prime} \\
& =\left(f_{x}^{\prime} g_{y}^{\prime}-f_{y}^{\prime} g_{x}^{\prime}\right)+\left(h_{x}^{\prime} g_{y}^{\prime}-h_{y}^{\prime} g_{x}^{\prime}\right)=[f, g]+[h, g] .
\end{aligned}
$$


Similarly, $[f, g+h]=[f, g]+[f, h]$.

$$
[f, f]=f_{x}^{\prime} f_{y}^{\prime}-f_{y}^{\prime} f_{x}^{\prime}=0 .
$$

$$
\begin{aligned}
& {[[f, g], h]+[[g, h], f]+[[h, f], g]} \\
& =\left[f_{x}^{\prime} g_{y}^{\prime}-f_{y}^{\prime} g_{x}^{\prime}, h\right]+\left[g_{x}^{\prime} h_{y}^{\prime}-g_{y}^{\prime} h_{x}^{\prime}, f\right]+\left[h_{x}^{\prime} f_{y}^{\prime}-h_{y}^{\prime} f_{x}^{\prime}, g\right] \\
& =\left(f_{x}^{\prime} g_{y}^{\prime}-f_{y}^{\prime} g_{x}^{\prime}\right)_{x}^{\prime} h_{y}^{\prime}-\left(f_{x}^{\prime} g_{y}^{\prime}-f_{y}^{\prime} g_{x}^{\prime}\right)_{y}^{\prime} h_{x}^{\prime} \\
& +\left(g_{x}^{\prime} h_{y}^{\prime}-g_{y}^{\prime} h_{x}^{\prime}\right)_{x}^{\prime} f_{y}^{\prime}-\left(g_{x}^{\prime} h_{y}^{\prime}-g_{y}^{\prime} h_{x}^{\prime}\right)_{y}^{\prime} f_{x}^{\prime} \\
& +\left(h_{x}^{\prime} f_{y}^{\prime}-h_{y}^{\prime} f_{x}^{\prime}\right)_{x}^{\prime} g_{y}^{\prime}-\left(h_{x}^{\prime} f_{y}^{\prime}-h_{y}^{\prime} f_{x}^{\prime}\right)_{y}^{\prime} g_{x}^{\prime} \\
& =\left(\left(f_{x}^{\prime} g_{y}^{\prime}\right)_{x}^{\prime}-\left(f_{y}^{\prime} g_{x}^{\prime}\right)_{x}^{\prime}\right) h_{y}^{\prime}-\left(\left(f_{x}^{\prime} g_{y}^{\prime}\right)_{y}^{\prime}-\left(f_{y}^{\prime} g_{x}^{\prime}\right)_{y}^{\prime}\right) h_{x}^{\prime} \\
& +\left(\left(g_{x}^{\prime} h_{y}^{\prime}\right)_{x}^{\prime}-\left(g_{y}^{\prime} h_{x}^{\prime}\right)_{x}^{\prime}\right) f_{y}^{\prime}-\left(\left(g_{x}^{\prime} h_{y}^{\prime}\right)_{y}^{\prime}-\left(g_{y}^{\prime} h_{x}^{\prime}\right)_{y}^{\prime}\right) f_{x}^{\prime} \\
& +\left(\left(h_{x}^{\prime} f_{y}^{\prime}\right)_{x}^{\prime}-\left(h_{y}^{\prime} f_{x}^{\prime}\right)_{x}^{\prime}\right) g_{y}^{\prime}-\left(\left(h_{x}^{\prime} f_{y}^{\prime}\right)_{y}^{\prime}-\left(h_{y}^{\prime} f_{x}^{\prime}\right)_{y}^{\prime}\right) g_{x}^{\prime} \\
& =\left(f_{x x}^{\prime \prime} g_{y}^{\prime}+f_{x}^{\prime} g_{y x}^{\prime \prime}-f_{y x}^{\prime \prime} g_{x}^{\prime}-f_{y}^{\prime} g_{x x}^{\prime \prime}\right) h_{y}^{\prime} \\
& -\left(f_{x y}^{\prime \prime} g_{y}^{\prime}+f_{x}^{\prime} g_{y y}^{\prime \prime}-f_{y y}^{\prime \prime} g_{x}^{\prime}-f_{y}^{\prime} g_{x y}^{\prime \prime}\right) h_{x}^{\prime} \\
& +\left(g_{x x}^{\prime \prime} h_{y}^{\prime}+g_{x}^{\prime} h_{y x}^{\prime \prime}-g_{y x}^{\prime \prime} h_{x}^{\prime}-g_{y}^{\prime} h_{x x}^{\prime \prime}\right) f_{y}^{\prime} \\
& -\left(g_{x y}^{\prime \prime} h_{y}^{\prime}+g_{x}^{\prime} h_{y y}^{\prime \prime}-g_{y y}^{\prime \prime} h_{x}^{\prime}-g_{y}^{\prime} h_{x y}^{\prime \prime}\right) f_{x}^{\prime} \\
& +\left(h_{x x}^{\prime \prime} f_{y}^{\prime}+h_{x}^{\prime} f_{y x}^{\prime \prime}-h_{y x}^{\prime \prime} f_{x}^{\prime}-h_{y}^{\prime} f_{x x}^{\prime \prime}\right) g_{y}^{\prime} \\
& -\left(h_{x y}^{\prime \prime} f_{y}^{\prime}+h_{x}^{\prime} f_{y y}^{\prime \prime}-h_{y y}^{\prime \prime} f_{x}^{\prime}-h_{y}^{\prime} f_{x y}^{\prime \prime}\right) g_{x}^{\prime} \\
& =f_{x x}^{\prime \prime} g_{y}^{\prime} h_{y}^{\prime}+f_{x}^{\prime} g_{y x}^{\prime \prime} h_{y}^{\prime}-f_{y x}^{\prime \prime} g_{x}^{\prime} h_{y}^{\prime}-f_{y}^{\prime} g_{x x}^{\prime \prime} h_{y}^{\prime} \\
& -f_{x y}^{\prime \prime} g_{y}^{\prime} h_{x}^{\prime}-f_{x}^{\prime} g_{y y}^{\prime \prime} h_{x}^{\prime}+f_{y y}^{\prime \prime} g_{x}^{\prime} h_{x}^{\prime}+f_{y}^{\prime} g_{x y}^{\prime \prime} h_{x}^{\prime} \\
& +g_{x x}^{\prime \prime} h_{y}^{\prime} f_{y}^{\prime}+g_{x}^{\prime} h_{y x}^{\prime \prime} f_{y}^{\prime}-g_{y x}^{\prime \prime} h_{x}^{\prime} f_{y}^{\prime}-g_{y}^{\prime} h_{x x}^{\prime \prime} f_{y}^{\prime} \\
& -g_{x y}^{\prime \prime} h_{y}^{\prime} f_{x}^{\prime}-g_{x}^{\prime} h_{y y}^{\prime \prime} f_{x}^{\prime}+g_{y y}^{\prime \prime} h_{x}^{\prime} f_{x}^{\prime}+g_{y}^{\prime} h_{x y}^{\prime \prime} f_{x}^{\prime} \\
& +h_{x x}^{\prime \prime} f_{y}^{\prime} g_{y}^{\prime}+h_{x}^{\prime} f_{y x}^{\prime \prime} g_{y}^{\prime}-h_{y x}^{\prime \prime} f_{x}^{\prime} g_{y}^{\prime}-h_{y}^{\prime} f_{x x}^{\prime \prime} g_{y}^{\prime} \\
& -h_{x y}^{\prime \prime} f_{y}^{\prime} g_{x}^{\prime}-h_{x}^{\prime} f_{y y}^{\prime \prime} g_{x}^{\prime}+h_{y y}^{\prime \prime} f_{x}^{\prime} g_{x}^{\prime}+h_{y}^{\prime} f_{x y}^{\prime \prime} g_{x}^{\prime} .
\end{aligned}
$$

Clearly, $f_{x y}^{\prime \prime}=f_{y x}^{\prime \prime}, g_{x y}^{\prime \prime}=g_{y x}^{\prime \prime}$ and $h_{x y}^{\prime \prime}=h_{y x}^{\prime \prime}$. Thus we obtain that

$$
[[f, g], h]+[[g, h], f]+[[h, f], g]=0 .
$$

Finally,

$$
\begin{aligned}
{[f g, h] } & =(f g)_{x}^{\prime} h_{y}^{\prime}-(f g)_{y}^{\prime} h_{x}^{\prime}=f_{x}^{\prime} g h_{y}^{\prime}+f g_{x}^{\prime} h_{y}^{\prime}-f_{y}^{\prime} g h_{x}^{\prime}-f g_{y}^{\prime} h_{x}^{\prime} \\
& =\left(f g_{x}^{\prime} h_{y}^{\prime}-f g_{y}^{\prime} h_{x}^{\prime}\right)+\left(f_{x}^{\prime} g h_{y}^{\prime}-f_{y}^{\prime} g h_{x}^{\prime}\right) \\
& =f\left(g_{x}^{\prime} h_{y}^{\prime}-g_{y}^{\prime} h_{x}^{\prime}\right)+g\left(f_{x}^{\prime} h_{y}^{\prime}-f_{y}^{\prime} h_{x}^{\prime}\right)=f[g, h]+g[f, h] .
\end{aligned}
$$

All these correspondences show that $P$ is a Poisson algebra. 
Example 3. Let $F$ be a field and $P=F\left[x_{1}, y_{1}, \ldots, x_{n}, y_{n}\right]$ be a polynomial algebra. Put $\left[x_{j}, y_{j}\right]=1,\left[x_{j}, y_{k}\right]=0$ whenever $j \neq k,\left[x_{j}, x_{k}\right]=$ $\left[y_{j}, y_{k}\right]=0$ for all $j, k, 1 \leqslant j, k \leqslant n$. Using Leibniz rule and the bilinearity of the operation, we define $\left[x_{j}^{m}, y_{k}^{s}\right],\left[x_{j}^{m}, x_{k}^{s}\right],\left[y_{j}^{m}, y_{k}^{s}\right]$. As we have proved $\left[x_{j}^{m}, y_{j}^{s}\right]=m s x_{j}^{m-1} y_{j}^{s-1}\left[x_{j}, y_{j}\right]$ for all positive integers $m, s$. In particular,

$$
\begin{gathered}
{\left[x_{j}^{m}, y_{j}^{s}\right]=m s x_{j}^{m-1} y_{j}^{s-1},} \\
{\left[x_{j}^{m}, y_{k}^{s}\right]=0}
\end{gathered}
$$

whenever $j \neq k$, and

$$
\left[x_{j}^{m}, x_{k}^{s}\right]=\left[y_{j}^{m}, y_{k}^{s}\right]=0
$$

for all $j, k, 1 \leqslant j, k \leqslant n$ and for all positive integers $m, s$. Further

$$
\begin{aligned}
{\left[x_{k}^{m_{k}} y_{k}^{s_{k}}, x_{j}^{t_{j}}\right] } & =x_{k}^{m_{k}}\left[y_{k}^{s_{k}}, x_{j}^{t_{j}}\right]+y_{k}^{s_{k}}\left[x_{k}^{m_{k}}, x_{j}^{t_{j}}\right] \\
& =y_{k}^{s_{k}}\left[x_{k}^{m_{k}}, x_{j}^{t_{j}}\right]-x_{k}^{m_{k}}\left[x_{j}^{t_{j}}, y_{k}^{s_{k}}\right] .
\end{aligned}
$$

If $k=j$, then

$$
\begin{aligned}
{\left[x_{k}^{m_{k}} y_{k}^{s_{k}}, x_{k}^{t_{k}}\right] } & =-x_{k}^{m_{k}}\left[x_{k}^{t_{k}}, y_{k}^{s_{k}}\right]=-s_{k} t_{k} x_{k}^{m_{k}} x_{k}^{t_{k}-1} y_{k}^{s_{k}-1} \\
& =-s_{k} t_{k} x_{k}^{m_{k}+t_{k}-1} y_{k}^{s_{k}-1} .
\end{aligned}
$$

If $k \neq j$, then $\left[x_{k}^{m_{k}} y_{k}^{s_{k}}, x_{j}^{t_{j}}\right]=0$.

At the next step we will find $\left[x_{k}^{m_{k}} y_{k}^{s_{k}}, y_{j}^{r_{j}}\right]$. We have

$$
\left[x_{k}^{m_{k}} y_{k}^{s_{k}}, y_{j}^{r_{j}}\right]=x_{k}^{m_{k}}\left[y_{k}^{s_{k}}, y_{j}^{r_{j}}\right]+y_{k}^{s_{k}}\left[x_{k}^{m_{k}}, y_{j}^{r_{j}}\right] .
$$

If $k=j$, then we obtain

$$
\left[x_{k}^{m_{k}} y_{k}^{s_{k}}, y_{k}^{r_{k}}\right]=y_{k}^{s_{k}}\left[x_{k}^{m_{k}}, y_{k}^{r_{k}}\right]=m_{k} r_{k} x_{k}^{m_{k}-1} y_{k}^{s_{k}+r_{k}-1}
$$

If $k \neq j$, then $\left[x_{k}^{m_{k}} y_{k}^{s_{k}}, y_{j}^{r_{j}}\right]=0$.

The next step is the finding of $\left[x_{k}^{m_{k}} y_{k}^{s_{k}}, x_{j}^{t_{j}} y_{j}^{r_{j}}\right]$. We have

$$
\left[x_{k}^{m_{k}} y_{k}^{s_{k}}, x_{j}^{t_{j}} y_{j}^{r_{j}}\right]=y_{j}^{r_{j}}\left[x_{k}^{m_{k}} y_{k}^{s_{k}}, x_{j}^{t_{j}}\right]+x_{j}^{t_{j}}\left[x_{k}^{m_{k}} y_{k}^{s_{k}}, y_{j}^{r_{j}}\right] .
$$

If $k=j$, then we obtain

$$
\begin{aligned}
{\left[x_{k}^{m_{k}} y_{k}^{s_{k}}, x_{k}^{t_{k}} y_{k}^{r_{k}}\right] } & =y_{k}^{r_{k}}\left(-s_{k} t_{k} x_{k}^{m_{k}+t_{k}-1} y_{k}^{s_{k}-1}\right)+x_{k}^{t_{k}}\left(m_{k} r_{k} x_{k}^{m_{k}-1} y_{k}^{s_{k}+r_{k}-1}\right) \\
& =\left(m_{k} r_{k}-s_{k} t_{k}\right) x_{k}^{m_{k}+t_{k}-1} y_{k}^{s_{k}+r_{k}-1} .
\end{aligned}
$$

If $k \neq j$, then $\left[x_{k}^{m_{k}} y_{k}^{s_{k}}, x_{j}^{t_{j}} y_{j}^{r_{j}}\right]=0$. 
Now we can find $\left[x_{1}^{m_{1}} y_{1}^{s_{1}} x_{2}^{m_{2}} y_{2}^{s_{2}} \ldots x_{n}^{m_{n}} y_{n}^{s_{n}}, x_{j}^{t_{j}} y_{j}^{r_{j}}\right]$. We have

$$
\begin{aligned}
& {\left[x_{1}^{m_{1}} y_{1}^{s_{1}} x_{2}^{m_{2}} y_{2}^{s_{2}} \ldots x_{n}^{m_{n}} y_{n}^{s_{n}}, x_{j}^{t_{j}} y_{j}^{r_{j}}\right]} \\
& =x_{1}^{m_{1}} y_{1}^{s_{1}} \ldots x_{j-1}^{m_{j-1}} y_{j-1}^{s_{j-1}} x_{j+1}^{m_{j+1}} y_{j+1}^{s_{j+1}} \ldots x_{n}^{m_{n}} y_{n}^{s_{n}}\left[x_{j}^{m_{j}} y_{j}^{s_{j}}, x_{j}^{t_{j}} y_{j}^{r_{j}}\right] \\
& =\left(m_{j} r_{j}-s_{j} t_{j}\right) x_{1}^{m_{1}} y_{1}^{s_{1}} \ldots x_{j-1}^{m_{j-1}} y_{j-1}^{s_{j-1}} \\
& \quad \quad \times x_{j}^{m_{j}+t_{j}-1} y_{j}^{s_{j}+r_{j}-1} x_{j+1}^{m_{j+1}} y_{j+1}^{s_{j+1}} \ldots x_{n}^{m_{n}} y_{n}^{s_{n}} .
\end{aligned}
$$

Finally,

$$
\begin{aligned}
& {\left[x_{1}^{m_{1}} y_{1}^{s_{1}} x_{2}^{m_{2}} y_{2}^{s_{2}} \ldots x_{n}^{m_{n}} y_{n}^{s_{n}}, x_{1}^{t_{1}} y_{1}^{r_{1}} x_{2}^{t_{2}} y_{2}^{r_{2}} \ldots x_{n}^{t_{n}} y_{n}^{r_{n}}\right]} \\
& =x_{2}^{t_{2}} y_{2}^{r_{2}} \ldots x_{n}^{t_{n}} y_{n}^{r_{n}}\left[x_{1}^{m_{1}} y_{1}^{s_{1}} x_{2}^{m_{2}} y_{2}^{s_{2}} \ldots x_{n}^{m_{n}} y_{n}^{s_{n}}, x_{1}^{t_{1}} y_{1}^{r_{1}}\right] \\
& \quad+x_{1}^{t_{1}} y_{1}^{r_{1}} x_{3}^{t_{3}} y_{3}^{r_{3}} \ldots x_{n}^{t_{n}} y_{n}^{r_{n}}\left[x_{1}^{m_{1}} y_{1}^{s_{1}} x_{2}^{m_{2}} y_{2}^{s_{2}} \ldots x_{n}^{m_{n}} y_{n}^{s_{n}}, x_{2}^{t_{2}} y_{2}^{r_{2}}\right]+\ldots \\
& \quad+x_{1}^{t_{1}} y_{1}^{r_{1}} \ldots x_{n-1}^{t_{n-1}} y_{n-1}^{r_{n-1}}\left[x_{1}^{m_{1}} y_{1}^{s_{1}} x_{2}^{m_{2}} y_{2}^{s_{2}} \ldots x_{n}^{m_{n}} y_{n}^{s_{n}}, x_{n}^{t_{n}} y_{n}^{r_{n}}\right] \\
& =\left(m_{1} r_{1}-s_{1} t_{1}\right) x_{1}^{m_{1}+t_{1}-1} y_{1}^{s_{1}+r_{1}-1} x_{2}^{m_{2}+t_{2}} y_{2}^{s_{2}+r_{2}} \ldots x_{n}^{m_{n}+t_{n}} y_{n}^{s_{n}+r_{n}} \\
& \quad+\left(m_{2} r_{2}-s_{2} t_{2}\right) x_{1}^{m_{1}+t_{1}} y_{1}^{s_{1}+r_{1}} x_{2}^{m_{2}+t_{2}-1} y_{2}^{s_{2}+r_{2}-1} \\
& \quad \quad \times x_{3}^{m_{3}+t_{3}} y_{3}^{s_{3}+r_{3}} \ldots x_{n}^{m_{n}+t_{n}} y_{n}^{s_{n}+r_{n}}+\ldots \\
& \quad+\left(m_{n} r_{n}-s_{n} t_{n}\right) x_{1}^{m_{1}+t_{1}} y_{1}^{s_{1}+r_{1}} \ldots x_{n-1}^{m_{n-1}+t_{n-1}} y_{n-1}^{s_{n-1}+r_{n-1}} \\
& \quad \quad \times x_{n}^{m_{n}+t_{n}-1} y_{n}^{s_{n}+r_{n}-1} \\
& \quad x_{1}^{m_{1}+t_{1}-1} y_{1}^{s_{1}+r_{1}-1} \ldots x_{n}^{m_{n}+t_{n}-1} y_{n}^{s_{n}+r_{n}-1}\left(\left(m_{1} r_{1}-s_{1} t_{1}\right) x_{2} y_{2} \ldots x_{n} y_{n}\right. \\
& \quad+\left(m_{2} r_{2}-s_{2} t_{2}\right) x_{1} y_{1} x_{3} y_{3} \ldots x_{n} y_{n}+\ldots \\
& \left.\quad+\left(m_{n} r_{n}-s_{n} t_{n}\right) x_{1} y_{1} \ldots x_{n-1} y_{n-1}\right) .
\end{aligned}
$$

Every element of $P$ is a linear combination of elements of the form $x_{1}^{m_{1}} y_{1}^{s_{1}} \ldots x_{n}^{m_{n}} y_{n}^{s_{n}}$, so taking into account the fact that the operation [, ] preserves addition, we get the value $[f, g]$ for every elements $f, g \in P$. In the resulting algebra, we constructed the operation $[$,$] in such a way$ that the Leibniz rule holds for it.

Let $S=\left\{x_{j}, y_{k} \mid 1 \leqslant j, k \leqslant n\right\}$. Consider all elements of the form

$$
J=[[u, v], w]+[[v, w], u]+[[w, u], v],
$$

where $u, v, w \in S$. By our definition, $[u, v]=0$ or $[u, v]=1$. It follows that every commutators $[[u, v], w],[[v, w], u],[[w, u], v]$ matches one of the following $[0,0],[0,1],[1,0],[1,1]$. Since all these commutators are equal to zero, $J=0$. Using now Proposition 1 , we obtain that $P$ is a Poisson algebra. 
Example 4. Let $F$ be a field and $L$ be a Lie algebra having a countable basis $\left\{a_{n} \mid n \in \mathbb{N}\right\}$. Let

$$
\left[a_{j}, a_{k}\right]=\sum_{n \in \mathbb{N}} \sigma_{j k, n} a_{n}
$$

$j, k \in \mathbb{N}$, where, as usual, it is assumed that all coefficients $\sigma_{j k, n}$, except for a finite number, are equal to 0 .

Let $P=F\left[x_{n} \mid n \in \mathbb{N}\right]$ be a polynomial algebra. Define an operation $[$,$] on P$ by the following rule. Put

$$
\left[x_{j}, x_{k}\right]=\sum_{n \in \mathbb{N}} \sigma_{j k, n} x_{n},
$$

$j, k \in \mathbb{N}$. Now we define the commutators $\left[x_{1}^{m_{1}} x_{2}^{m_{2}} \ldots x_{n}^{m_{n}}, x_{1}^{t_{1}} x_{2}^{t_{2}} \ldots x_{k}^{t_{k}}\right]$ in the same way as it was done in the construction of the previous example. The computations here are more cumbersome, so we will not present them here. Every element of algebra $P$ is a linear combination of the elements of the type $x_{1}^{m_{1}} \ldots x_{n}^{m_{n}}$, so taking into account the fact that the operation $[$,$] preserves addition, we get the value of [f, g]$ for all elements $f, g \in P$. In the resulting algebra, we constructed the operation $[$,$] exactly in$ such a way that it holds Leibniz rule. Since the elements $\left\{a_{n} \mid n \in \mathbb{N}\right\}$ constitute a basis of the Lie algebra, for which the Jacobi identity holds. This implies that the Jacobi identity holds for the elements $\left\{x_{n} \mid n \in \mathbb{N}\right\}$. These elements generate $P$ as an associative algebra. Proposition 1 implies that $P$ is a Poisson algebra.

Proposition 4. Let $P$ be a Poisson algebra over a field $F$. Suppose that $A$ is a non-abelian subalgebra of $P$. If $A$ is nilpotent, then $A$ contains zero divisors.

Proof. Let

$$
A=\gamma_{1}(A) \geqslant \gamma_{2}(A) \geqslant \ldots \geqslant \gamma_{n}(A) \geqslant \gamma_{n+1}(A)=\langle 0\rangle
$$

be the lower central series of $A$. Since $A$ is not abelian, $Z=\gamma_{n}(A) \neq$ $\gamma_{n-1}(A)=S$. It follows that there are elements $x \in S, y \in A$ such that $[x, y] \neq 0$. Since $x \in S=\gamma_{n-1}(A),[x, y] \in\left[\gamma_{n-1}(A), A\right]=\gamma_{n}(A)=$ $Z$. An inclusion $Z \leqslant \zeta(A)$ shows that $[[x, y], y]=0$. In a similar way, $\left[\left[x^{2}, y\right], y\right]=0$.

Suppose first that $F$ has a characteristic 0 . Then

$$
0=\left[\left[x^{2}, y\right], y\right]=[2 x[x, y], y]=2[x, y][x, y]+2 x[[x, y], y]=2[x, y]^{2} .
$$

Since $\operatorname{char}(F) \neq 2$, it follows that $[x, y]^{2}=0$. 
Suppose now that $F$ has a prime characteristic $p$. As we have seen above, $\left[x^{n}, y\right]=n x^{n-1}[x, y]$ for arbitrary positive integer $n$. If there exists a positive integer $n$ such that $x^{n}=0$, then all is proved. Therefore we can assume, that $x^{n} \neq 0$ for every positive integer $n$. If we suppose that there exists a positive integer $n$ such that $\operatorname{GCD}(n, p)=1$ and $\left[x^{n}, y\right]=0$, then $x^{n-1}[x, y]=0$, and again all is proved. Thus we will suppose that $\left[x^{n}, y\right] \neq 0$ for each positive integer $n$ such that $\operatorname{GCD}(n, p)=1$. Choose a positive integer $k$ such that $k>p+1$ and $\operatorname{GCD}(k, p)=1$, then

$$
\begin{gathered}
0=\left[\left[x^{k}, y\right], y\right]=\left[k x^{k-1}[x, y], y\right]=k[x, y]\left[x^{k-1}, y\right]+k x^{k-1}[[x, y], y]= \\
=k[x, y]\left[x^{k-1}, y\right] .
\end{gathered}
$$

Since $k-1>p$, as we have noted above $\left[x^{k-1}, y\right] \neq 0$. Thus the product of two non-zero elements $[x, y]$ and $\left[x^{k-1}, y\right]$ is zero, and at last all is proved.

The Poisson algebra $P$ is called locally nilpotent, if every finitely generated subalgebra of $P$ is nilpotent.

Corollary 1. Let $P$ be a Poisson algebra over a field $F$. Suppose that $A$ is a locally nilpotent subalgebra of $P$. If $A$ does not contain zero divisors, then $A$ is abelian.

Proof. Indeed, suppose that $A$ is not abelian. Then $A$ has the elements $x, y$ such that $[x, y] \neq 0$. Let $S$ be a subalgebra of $A$, generated by the elements $x, y$. Then this subalgebra is not abelian. Then Proposition 4 shows that $S$, being nilpotent, must contain zero divisors, and we obtain a contradiction. This contradiction shows that $A$ is abelian.

Proposition 5. Let $P$ be a Poisson algebra over a field $F$. Suppose that $\operatorname{char}(F)=p$ is a prime and $F^{p}=F$. If $S$ is a subalgebra of $P$, then the subset $S^{p}=\left\{x^{p} \mid x \in S\right\}$ is a subalgebra of $P$, moreover, $S^{p} \leqslant \zeta(P)$.

Proof. Let $x \in S^{p}$, and $\lambda$ is an arbitrary element of a field $F$. Then $x=a^{p}$ for some element $a \in S$. Equality $F^{p}=F$ implies that $\lambda=\mu^{p}$ for some element $\mu \in F$. Then $\lambda x=\mu^{p} a^{p}=(\mu a)^{p} \in S^{p}$.

If $y$ is another element of $S^{p}$, then $y=b^{p}$ for some element $b \in S$. If $p=2$, then $-y=y$ and $x-y=x+y=a^{p}+b^{p}=(a+b)^{p} \in S^{p}$. If $p>2$, then $x-y=a^{p}-b^{p}=(a-b)^{p} \in S^{p}$. Further $x y=a^{p} b^{p}=(a b)^{p} \in S^{p}$.

Let $c$ be an arbitrary element of $P$ and $a$ be an arbitrary element of $S$. As we have proved $\left[a^{p}, c\right]=p a^{p-1}[a, c]=0$. It follows that $S^{p} \leqslant \zeta(P)$. 
Corollary 2. Let $P$ be a Poisson algebra over a field $F$. Suppose that $\operatorname{char}(F)=p$ is a prime and $F^{p}=F$. Then the subset $P^{p}=\left\{x^{p} \mid x \in P\right\}$ is a subalgebra of $P$, moreover, $P^{p} \leqslant \zeta(P)$.

Corollary 3. Let $P$ be a Poisson algebra over a field $F$. Suppose that $\operatorname{char}(F)=p$ is a prime and $F^{p}=F$. If $S$ is a finite-dimensional subalgebra of $P$ and $S$ does not contain zero divisors, then $S \leqslant \zeta(P)$.

Proof. Consider the mapping $f: S \rightarrow S$ defined by the rule: $f(x)=x^{p}$ for all $x \in S$. We have $\operatorname{Im}(f)=S^{p}$. By Proposition $5, S^{p}$ is a subalgebra of $P$. Since $S$ does not contains zero divisors, $\operatorname{Ker}(f)=\langle 0\rangle$. Thus $S \cong_{F} S^{p}$. In particular, $\operatorname{dim}_{F}(S)=\operatorname{dim}_{F}\left(S^{p}\right)$. It follows that $S=S^{p}$. Proposition 5 proves inclusion $S=S^{p} \leqslant \zeta(P)$.

Corollary 4. Let $P$ be a Poisson algebra over a field $F$. Suppose that $\operatorname{char}(F)=p$ is a prime and $F^{p}=F$. If $P$ is finite-dimensional and $P$ does not contain zero divisors, then $P$ is abelian.

We note that every finite field $F$ of characteristic $p$ satisfies $F^{p}=F$. It implies that every locally finite field $F$ of characteristic $p$ satisfies $F^{p}=F$. Thus, we obtain

Corollary 5. Let $P$ be a Poisson algebra over a finite field $F$. If $P$ is finite-dimensional and $P$ does not contain zero divisors, then $P$ is abelian.

Corollary 6. Let $P$ be a Poisson algebra over a locally finite field $F$. If $P$ is finite-dimensional and $P$ does not contain zero divisors, then $P$ is abelian.

Corollary 7. Let $P$ be a Poisson algebra over a field F. Suppose that $\operatorname{char}(F)=p$ is a prime and $F^{p}=F$. If $S$ is a locally (finite-dimensional) subalgebra of $P$ and $S$ does not contain zero divisors, then $S \leqslant \zeta(P)$.

Proof. Indeed, every finitely generated subalgebra $A$ of $S$ has finite dimension. Then Corollary 3 implies that $A \leqslant \zeta(P)$. Since it is true for every finitely generated subalgebra of $S, S \leqslant \zeta(P)$.

Corollary 8. Let $P$ be a Poisson algebra over a field $F$. Suppose that $\operatorname{char}(F)=p$ is a prime and $F^{p}=F$. If $P$ is locally (finite-dimensional) and $P$ does not contain zero divisors, then $P$ is abelian.

Corollary 9. Let $P$ be a Poisson algebra over a finite field $F$. If $P$ is locally (finite-dimensional) and $P$ does not contain zero divisors, then $P$ is abelian.

Corollary 10. Let $P$ be a Poisson algebra over a locally finite field $F$. If $P$ is locally (finite-dimensional) and $P$ does not contain zero divisors, then $P$ is abelian. 


\section{The Poisson algebras that are finite-dimensional over the upper hypercenter}

Lemma 1. Let $L$ be a Lie algebra over a field $F$. If $j, k$ are positive integers such that $k \geqslant j$, then $\left[\gamma_{j}(L), \zeta_{k}(L)\right] \leqslant \zeta_{k-j}(L)$.

Proof. We will use induction on $j$. For $j=1$ we have

$$
\left[\gamma_{1}(L), \zeta_{k}(L)\right]=\left[L, \zeta_{k}(L)\right] \leqslant \zeta_{k-1}(L)
$$

Suppose now that $j>1$. We have already proved the inclusions

$$
\left[\gamma_{m}(L), \zeta_{k}(L)\right] \leqslant \zeta_{k-m}(L)
$$

for all $m<j$. Choose the arbitrary elements $x \in L, y \in \gamma_{j-1}(L), z \in \zeta_{k}(L)$. We have

$$
[[x, y], z]=[x,[y, z]]-[y,[x, z]] .
$$

Since $[y, z] \in\left[\gamma_{j-1}(L), \zeta_{k}(L)\right]$, the induction hypothesis implies that $[y, z] \in \zeta_{k-j+1}(L)$, so that, $[x,[y, z]] \in\left[L, \zeta_{k-j+1}(L)\right] \leqslant \zeta_{k-j}(L)$. Further,

$$
[y,[x, z]] \in\left[\gamma_{j-1}(L),\left[L, \zeta_{k}(L)\right]\right] \leqslant\left[\gamma_{j-1}(L), \zeta_{k-1}(L)\right] .
$$

Since $k-1>j-1$, using the induction hypothesis, we obtain that

$$
\left[\gamma_{j-1}(L), \zeta_{k-1}(L)\right] \leqslant \zeta_{k-1-j+1}(L)=\zeta_{k-j}(L) .
$$

It follows that $\left[\gamma_{j}(L), \zeta_{k}(L)\right] \leqslant \zeta_{k-j}(L)$.

Lemma 2. Let $L$ be a Lie algebra over a field $F$. Suppose that the factoralgebra $L / \zeta_{n}(L)$ has a finite codimension d and let $\left\{e_{1}+\zeta_{n}(L), \ldots, e_{d}+\right.$ $\left.\zeta_{n}(L)\right\}$ be a basis of $L / \zeta_{n}(L)$. Then $\gamma_{n+1}(L)$ generated by the elements $\left[u_{1}, \ldots, u_{n}, u_{n+1}\right]$ where $u_{j} \in\left\{e_{1}, \ldots, e_{d}\right\}, 1 \leqslant j \leqslant n+1$.

Proof. We use induction by $n$. Suppose first that $n=1$ and put $Z=\zeta(L)$. Then for every element $x \in L$ we have $x=\lambda_{1} e_{1}+\ldots+\lambda_{d} e_{d}+z_{x}$ for suitable coefficients $\lambda_{1}, \ldots, \lambda_{d} \in F$ and element $z_{x} \in Z$.

Let $y=\mu_{1} e_{1}+\ldots+\mu_{d} e_{d}+z_{y}, \mu_{1}, \ldots, \mu_{d} \in F, z_{y} \in Z$. Then

$$
\begin{aligned}
{[x, y] } & =\left[\lambda_{1} e_{1}+\ldots+\lambda_{d} e_{d}+z_{x}, \mu_{1} e_{1}+\ldots+\mu_{d} e_{d}+z_{y}\right] \\
& =\sum_{1 \leqslant j, k \leqslant d} \lambda_{j} \mu_{k}\left[e_{j}, e_{k}\right] .
\end{aligned}
$$

As we can see, $\gamma_{2}(L)=[L, L]$ is a subalgebra generates by the elements $\left[e_{j}, e_{k}\right], 1 \leqslant j, k \leqslant d$. More precisely, since $\left[e_{j}, e_{k}\right]=-\left[e_{k}, e_{j}\right]$ 
and $\left[e_{j}, e_{j}\right]=0,1 \leqslant j, k \leqslant d, \gamma_{2}(L)$ generates by the elements $\left[e_{j}, e_{k}\right]$ where $1 \leqslant j<k \leqslant d$.

Suppose now that $n>1$ and that we have already proved that an ideal $\gamma_{n}(L / Z)$ generated by the elements $\left[u_{1}+Z, \ldots, u_{n}+Z\right]=\left[u_{1}, \ldots, u_{n}\right]+Z$, where $u_{j} \in\left\{e_{1}, \ldots, e_{d}\right\}, 1 \leqslant j \leqslant n$. Let $x$ be an arbitrary element of $L$ and $y$ be an arbitrary element of $\gamma_{n}(L)$. Then $y+Z \in \gamma_{n}(L)+Z=\gamma_{n}(L / Z)$, so that $y=\left[u_{1}, \ldots, u_{n}\right]+z$ for some element $z \in Z$. For element $x$ we obtain $x=\lambda_{1} e_{1}+\ldots+\lambda_{d} e_{d}+c_{x}$ for suitable coefficients $\lambda_{1}, \ldots, \lambda_{d} \in F$ and element $c_{x} \in \zeta_{n}(L)$.

We have

$$
\begin{aligned}
{[x, y] } & =\left[\lambda_{1} e_{1}+\ldots+\lambda_{d} e_{d}+c_{x},\left[u_{1}, \ldots, u_{n}\right]+z\right] \\
& =\lambda_{1}\left[e_{1},\left[u_{1}, \ldots, u_{n}\right]\right]+\ldots+\lambda_{d}\left[e_{d},\left[u_{1}, \ldots, u_{n}\right]\right]+\left[c_{x},\left[u_{1}, \ldots, u_{n}\right]\right] .
\end{aligned}
$$

Lemma 1 implies that $\left[c_{x},\left[u_{1}, \ldots, u_{n}\right]\right]=0$, and the result is proved.

Proof of Theorem $A$. Put $Z=\zeta(P)$. Then $P=Z \oplus A$ for some subspace $A$ of $P$. Choose a basis $\left\{e_{1}, \ldots, e_{d}\right\}$ in the subspace $A$. Then for every element $x \in P$ we have $x=\lambda_{1} e_{1}+\ldots+\lambda_{d} e_{d}+z_{x}$ for suitable coefficients $\lambda_{1}, \ldots, \lambda_{d} \in F$ and element $z_{x} \in Z$.

A subspace $[P, P]$ is an ideal of a Lie algebra $P(+,[]$,$) . Lemma 2$ shows that $[P, P]$ generates as a subspace by the elements $\left[e_{j}, e_{k}\right]$ where $1 \leqslant j, k \leqslant d$. More precisely, since $\left[e_{j}, e_{k}\right]=-\left[e_{k}, e_{j}\right]$ and $\left[e_{j}, e_{j}\right]=0$, $1 \leqslant j, k \leqslant d$, the subalgebra $[P, P]$ is generated by the elements $\left[e_{j}, e_{k}\right]$ where $1 \leqslant j<k \leqslant d$.

Consider an ideal $K$ of an associative algebra $P(+, \cdot)$ generated by $[P, P]$. Every its element has a form $a_{1} x_{1}+\ldots+a_{r} x_{r}$ where $a_{1}, \ldots, a_{r} \in$ $[P, P], x_{1}, \ldots, x_{r}$ are the arbitrary elements of $P$. Let $x$ be an arbitrary element of $P, x=\lambda_{1} e_{1}+\ldots+\lambda_{d} e_{d}+z_{x}$ where $\lambda_{1}, \ldots, \lambda_{d} \in F$ and $z_{x} \in Z$. We have

$$
\begin{aligned}
{\left[e_{j}, e_{k}\right] x } & =\left[e_{j}, e_{k}\right]\left(\lambda_{1} e_{1}+\ldots+\lambda_{d} e_{d}+z_{x}\right) \\
& =\lambda_{1} e_{1}\left[e_{j}, e_{k}\right]+\ldots+\lambda_{d} e_{d}\left[e_{j}, e_{k}\right]+z_{x}\left[e_{j}, e_{k}\right] .
\end{aligned}
$$

Using Leibniz rule we obtain

$$
\left[e_{j}, e_{k} z_{x}\right]=e_{k}\left[e_{j}, z_{x}\right]+z_{x}\left[e_{j}, e_{k}\right]=z_{x}\left[e_{j}, e_{k}\right] .
$$

For element $e_{k} z_{x}$ we have the decomposition $e_{k} z_{x}=\nu_{1} e_{1}+\ldots+\nu_{d} e_{d}+z_{x, k}$. Therefore

$$
\left[e_{j}, e_{k} z_{x}\right]=\left[e_{j}, \nu_{1} e_{1}+\ldots+\nu_{d} e_{d}+z_{x, k}\right]=\nu_{1}\left[e_{j}, e_{1}\right]+\ldots+\nu_{d}\left[e_{j}, e_{d}\right] .
$$


These equalities show that $K$ as a vector space is generated by the elements $\left[e_{j}, e_{k}\right], e_{s}\left[e_{j}, e_{k}\right], 1 \leqslant s \leqslant d, 1 \leqslant j<k \leqslant d$. It follows that $K$ has a dimension at most

$$
\frac{1}{2} d(d-1)+d\left(\frac{1}{2} d(d-1)\right)=\frac{1}{2} d(d-1)(d+1)=\frac{1}{2} d\left(d^{2}-1\right) .
$$

The inclusion $[K, K] \leqslant[P, P] \leqslant K$ shows that $K$ is a subalgebra of $P$. Moreover, $[K, P] \leqslant[P, P] \leqslant K$, so that $K$ is an ideal of Lie algebra $P(+,[]$,$) .$

Corollary 11. Let $P$ be a Poisson algebra over a field $F$. Suppose that $\operatorname{char}(F)=p$ is a prime and $F^{p}=F$. If $P$ is finite-dimensional over the center and $P$ does not contain zero divisors, then $P$ is abelian.

Proof. Theorem A implies that $P$ includes a finite-dimensional ideal $A$ such that $P / A$ is abelian. By Corollary $3, A \leqslant \zeta(P)$. Hence, $P$ is nilpotent. Using now Corollary 1 we obtain that $P$ must be abelian.

Corollary 12. Let $P$ be a Poisson algebra over a finite field $F$. If $P$ is finite-dimensional over the center and $P$ does not contain zero divisors, then $P$ is abelian.

Corollary 13. Let $P$ be a Poisson algebra over a locally finite field $F$. If $P$ is finite-dimensional over the center and $P$ does not contain zero divisors, then $P$ is abelian.

Proof of Theorem $B$. Put $Z=\zeta_{n}(P)$. Then $P=Z \oplus A$ for some subspace $A$ of $P$. Choose an arbitrary basis $\left\{e_{1}, \ldots, e_{d}\right\}$ in the subspace $A$. Then for every element $x \in P$ we have

$$
x=\lambda_{1} e_{1}+\ldots+\lambda_{d} e_{d}+z_{x}
$$

for suitable coefficients $\lambda_{1}, \ldots, \lambda_{d} \in F$ and element $z_{x} \in Z$.

A subspace $\gamma_{n+1}(P)$ is an ideal of a Lie algebra $P(+,[]$,$) . Lemma 2$ shows that $\gamma_{n+1}(P)$ generates as a subspace by $\left[u_{1}, \ldots, u_{n}, u_{n+1}\right]$ where $u_{j} \in\left\{e_{1}, \ldots, e_{d}\right\}, 1 \leqslant j \leqslant n+1$. In particular, $\operatorname{dim}_{F}\left(\gamma_{n+1}(P)\right) \leqslant d^{n+1}$.

Consider an ideal $K$ of an associative algebra $P(+, \cdot)$, generated by $\gamma_{n+1}(P)$. Every its element has a form $a_{1} x_{1}+\ldots+a_{r} x_{r}$ where $a_{1}, \ldots, a_{r} \in$ $\gamma_{n+1}(P), x_{1}, \ldots, x_{r}$ are the arbitrary elements of $P$. Let $x$ be an arbitrary element of $P, x=\lambda_{1} e_{1}+\ldots+\lambda_{d} e_{d}+z_{x}$ where $\lambda_{1}, \ldots, \lambda_{d} \in F$ and $z_{x} \in Z$. We have

$$
\begin{aligned}
& {\left[u_{1}, \ldots, u_{n+1}\right] x=\left[u_{1}, \ldots, u_{n+1}\right]\left(\lambda_{1} e_{1}+\ldots+\lambda_{d} e_{d}+z_{x}\right)} \\
& \quad=\lambda_{1} e_{1}\left[u_{1}, \ldots, u_{n+1}\right]+\ldots+\lambda_{d} e_{d}\left[u_{1}, \ldots, u_{n+1}\right]+z_{x}\left[u_{1}, \ldots, u_{n+1}\right] .
\end{aligned}
$$


Using Leibniz rule we obtain

$$
\left[\left[u_{1}, \ldots, u_{n}\right], u_{n+1} z_{x}\right]=u_{n+1}\left[\left[u_{1}, \ldots, u_{n}\right], z_{x}\right]+z_{x}\left[\left[u_{1}, \ldots, u_{n}\right], u_{n+1}\right] .
$$

Lemma 1 implies that $\left[\left[u_{1}, \ldots, u_{n}\right], z_{x}\right]=0$. Hence

$$
z_{x}\left[u_{1}, \ldots, u_{n+1}\right]=\left[\left[u_{1}, \ldots, u_{n}\right], u_{n+1} z_{x}\right] .
$$

For element $u_{n+1} z_{x}$ we have decomposition $u_{n+1} z_{x}=\nu_{1} e_{1}+\ldots+\nu_{d} e_{d}+z_{x, n}$ where again $z_{x, n} \in Z$, therefore

$$
\begin{aligned}
& {\left[\left[u_{1}, \ldots, u_{n}\right], u_{n+1} z_{x}\right]=\left[\left[u_{1}, \ldots, u_{n}\right], \nu_{1} e_{1}+\ldots+\nu_{d} e_{d}+z_{x, n}\right]} \\
& \quad=\nu_{1}\left[\left[u_{1}, \ldots, u_{n}\right], e_{1}\right]+\ldots+\nu_{d}\left[\left[u_{1}, \ldots, u_{n}\right], e_{d}\right]+\left[\left[u_{1}, \ldots, u_{n}\right], z_{x, n}\right]
\end{aligned}
$$

By Lemma $1,\left[\left[u_{1}, \ldots, u_{n}\right], z_{x, n}\right]=0$. It follows that $z_{x}\left[u_{1}, \ldots, u_{n+1}\right] \in$ $\gamma_{n+1}(P)$. Thus we can see that $K$ as a vector space is generated by the elements $\left[u_{1}, \ldots, u_{n}, u_{n+1}\right]$ and $e_{s}\left[u_{1}, \ldots, u_{n}, u_{n+1}\right], u_{j} \in\left\{e_{1}, \ldots, e_{d}\right\}$, $1 \leqslant j \leqslant n+1,1 \leqslant s \leqslant d$. It follows that $\operatorname{dim}_{F}(K) \leqslant d^{n+1}+d d^{n+1}=$ $d^{n+1}(1+d)$.

Let $x$ be an arbitrary element of $P, x=\lambda_{1} e_{1}+\ldots+\lambda_{d} e_{d}+z_{x}$ where $\lambda_{1}, \ldots, \lambda_{d} \in F$ and $z_{x} \in Z$. Since $\gamma_{n+1}(P)$ is an ideal of Lie algebra $P(+,[]),,\left[\left[u_{1}, \ldots, u_{n+1}\right], x\right] \in \gamma_{n+1}(P)$. Furthermore

$$
\begin{aligned}
& {\left[e_{s}\left[u_{1}, \ldots, u_{n+1}\right], x\right]=\left[e_{s}\left[u_{1}, \ldots, u_{n+1}\right], \lambda_{1} e_{1}+\ldots+\lambda_{d} e_{d}+z_{x}\right]} \\
& =\lambda_{1}\left[e_{s}\left[u_{1}, \ldots, u_{n+1}\right], e_{1}\right]+\ldots+\lambda_{d}\left[e_{s}\left[u_{1}, \ldots, u_{n+1}\right], e_{d}\right] \\
& \quad+\left[e_{s}\left[u_{1}, \ldots, u_{n+1}\right], z_{x}\right] .
\end{aligned}
$$

We have

$$
\left[e_{s}\left[u_{1}, \ldots, u_{n+1}\right], e_{j}\right]=\left[u_{1}, \ldots, u_{n+1}\right]\left[e_{s}, e_{j}\right]+e_{s}\left[\left[u_{1}, \ldots, u_{n+1}\right], e_{j}\right] .
$$

We note that $\left[u_{1}, \ldots, u_{n+1}\right]\left[e_{s}, e_{j}\right] \in K,\left[\left[u_{1}, \ldots, u_{n+1}\right], e_{j}\right] \in \gamma_{n+1}(P)$, so that $e_{s}\left[\left[u_{1}, \ldots, u_{n+1}\right], e_{j}\right] \in K$. Thus $\left[e_{s}\left[u_{1}, \ldots, u_{n+1}\right], e_{j}\right] \in K$. Now we have

$$
\left[e_{s}\left[u_{1}, \ldots, u_{n+1}\right], z_{x}\right]=\left[u_{1}, \ldots, u_{n+1}\right]\left[e_{s}, z_{x}\right]+e_{s}\left[\left[u_{1}, \ldots, u_{n+1}\right], z_{x}\right] .
$$

We note that $\left[u_{1}, \ldots, u_{n+1}\right]\left[e_{s}, z_{x}\right] \in K$ and $\left[\left[u_{1}, \ldots, u_{n+1}\right], z_{x}\right]=0$ by Lemma 1. Hence $\left[e_{s}\left[u_{1}, \ldots, u_{n+1}\right], z_{x}\right] \in K$. These inclusion shows that $K$ also is an ideal of Lie algebra $P(+,[]$,$) . Then K$ is an ideal of Poisson algebra $P$. Finally, inclusion $\gamma_{n+1}(P) \leqslant K$ shows that factor-algebra $P / K$ is nilpotent of nilpotency class at most $n$. 
Proof of Corollary B1. Theorem B implies that an algebra $P$ includes a finite-dimensional ideal $A$ such that $P / A$ is nilpotent. By Corollary 3, $A \leqslant \zeta(P)$. Hence, $P$ is nilpotent. Using Corollary 1 we obtain that $P$ must be abelian.

Now we present our last result.

Theorem 1. Let $P$ be a finitely generated Poisson algebra over a field $F$ and $K$ be an ideal of $P$. If $K$ has a finite codimension, then $K$ is finitely generated as an ideal.

Proof. Let $M=\left\{a_{1}, \ldots, a_{n}\right\}$ be a finite subset generated $P$, and let $B$ be a subspace of $P$ such that $P=B \oplus K$. Let $\operatorname{codim}_{F}(K)=d$. Then $\operatorname{dim}_{F}(B)=d$. Choose in $B$ a basis $\left\{b_{1}, \ldots, b_{d}\right\}$. Denote by $\operatorname{pr}_{B}$ (respectively $\operatorname{pr}_{K}$ ) the canonical projection of $P$ on $B$ (respectively on $K$ ). Let $E$ be the ideal, generated by the elements

$$
\left\{\operatorname{pr}_{K}\left(a_{j}\right), \operatorname{pr}_{K}\left(\left[a_{j}, b_{m}\right]\right), \operatorname{pr}_{K}\left(a_{j} b_{m}\right) \mid 1 \leqslant j \leqslant n, 1 \leqslant m \leqslant d\right\} .
$$

By this choice, $K$ includes $E$, and $E$ is a finitely generated as an ideal of $P$. If $x$ is an arbitrary element of $E+B$, then $x=u+b$ where $u \in E, b \in B$. Furthermore, $b=\alpha_{1} b_{1}+\ldots+\alpha_{d} b_{d}$ for suitable elements $\alpha_{1}, \ldots, \alpha_{d} \in F$. We have

$$
\begin{gathered}
{\left[b, a_{j}\right]=\left[\alpha_{1} b_{1}+\ldots+\alpha_{d} b_{d}, a_{j}\right]=\alpha_{1}\left[b_{1}, a_{j}\right]+\ldots+\alpha_{d}\left[b_{d}, a_{j}\right]} \\
=\alpha_{1}\left(\operatorname{pr}_{K}\left(\left[b_{1}, a_{j}\right]\right)+\operatorname{pr}_{B}\left(\left[b_{1}, a_{j}\right]\right)\right)+\ldots \\
\quad+\alpha_{d}\left(\operatorname{pr}_{K}\left(\left[b_{d}, a_{j}\right]\right)+\operatorname{pr}_{B}\left(\left[b_{d}, a_{j}\right]\right)\right) \\
=\alpha_{1} \operatorname{pr}_{K}\left(\left[b_{1}, a_{j}\right]\right)+\ldots+\alpha_{d} \operatorname{pr}_{K}\left(\left[b_{d}, a_{j}\right]\right) \\
\quad+\alpha_{1} \operatorname{pr}_{B}\left(\left[b_{1}, a_{j}\right]\right)+\ldots+\alpha_{d} \operatorname{pr}_{B}\left(\left[b_{d}, a_{j}\right]\right), \\
b a_{j}=\left(\alpha_{1} b_{1}+\ldots+\alpha_{d} b_{d}\right) a_{j}=\alpha_{1}\left(b_{1} a_{j}\right)+\ldots+\alpha_{d}\left(b_{d} a_{j}\right) \\
=\alpha_{1}\left(\operatorname{pr}_{K}\left(b_{1} a_{j}\right)+\operatorname{pr}_{B}\left(b_{1} a_{j}\right)\right)+\ldots+\alpha_{d}\left(\operatorname{pr}_{K}\left(b_{d} a_{j}\right)+\operatorname{pr}_{B}\left(b_{d} a_{j}\right)\right) \\
=\alpha_{1} \operatorname{pr}_{K}\left(b_{1} a_{j}\right)+\ldots+\alpha_{d} \operatorname{pr}_{K}\left(b_{d} a_{j}\right)+\alpha_{1} \operatorname{pr}_{B}\left(b_{1} a_{j}\right)+\ldots+\alpha_{d} \operatorname{pr}_{B}\left(b_{d} a_{j}\right) .
\end{gathered}
$$

The elements

$$
\sum_{1 \leqslant m \leqslant d}\left(\alpha_{m} \operatorname{pr}_{K}\left(\left[b_{m}, a_{j}\right]\right)+\alpha_{m} \operatorname{pr}_{B}\left(\left[b_{m}, a_{j}\right]\right)\right)
$$

and

$$
\sum_{1 \leqslant m \leqslant d}\left(\alpha_{m} \operatorname{pr}_{K}\left(b_{m} a_{j}\right)+\alpha_{m} \operatorname{pr}_{B}\left(b_{m} a_{j}\right)\right)
$$

clearly belong to $E+B$. It follows that $E+B$ is an ideal of $P$. The equality $a_{j}=\operatorname{pr}_{K}\left(a_{j}\right)+\operatorname{pr}_{B}\left(a_{j}\right)$ implies that $a_{j} \in E+B, 1 \leqslant j \leqslant n$. It 
follows that $E+B=P=K+B$. The inclusion $E \leqslant K$ and the equation $K \cap B=\langle 0\rangle$ imply that $K=E$. In particular, $K$ is a finitely generated as an ideal.

\section{References}

[1] V. I. Arnold, Mathematical Methods of Classical Mechanics, Graduate Texts Math. 60, Springer-Verlag, New York - Heidelberg, 1978.

[2] C. J. Atkin, A note on the algebra of Poisson brackets, Math. Proc. Cambridge Philos. Soc., 96(1), 1984, 45-60.

[3] R. Baer, Endlichkeitskriterien für Kommutatorgruppen, Math. Ann., 124, 1952, 161-177.

[4] F. Bayen, M. Flato, C. Fronsdal, A. Lichnerowicz, D. Sternheimer, Deformation theory and quantization. I. Deformations of symplectic structures, Ann. Physics, 111(1), 1978, 61-110.

[5] F. Bayen, M. Flato, C. Fronsdal, A. Lichnerowicz, D. Sternheimer, Deformation theory and quantization. II. Physical applications, Ann. Physics, 111(1), 1978, 111-151.

[6] J. Bell, S. Launois, O. L. Sánchez, R. Moosa, Poisson algebras via model theory and differential-algebraic geometry, J. Eur. Math. Soc., 19(7), 2017, 2019-2049.

[7] F. A. Berezin, Some remarks about the associated envelope of a Lie algebra, Funct. Anal. Appl., 1(2), 1967, 91-102.

[8] K. H. Bhaskara, K. Viswanath, Poisson algebras and Poisson manifolds, Longman, 1988.

[9] J. Braconnier, Algebres de Poisson, C.R. Acad. Sci., A, 284(21), 1977, 1345-1348.

[10] A. J. Calderón Martín, On extended graded Poisson algebras, Linear Algebra Appl., 439(4), 2013, 879-892.

[11] V. Chari, A. Pressley, A Guide to Quantum Groups, Cambridge University Press, Cambridge, 1994.

[12] P. A. M. Dirac, Lectures on Quantum Mechanics, Belfer Grad. Sch. Sci., Yeshiva University, New York, 1964.

[13] V. G. Drinfeld, Quantum groups, Zap. Nauchn. Sem. LOMI, 155, 1986, 18-49; J. Soviet Math., 41(2), 1988, 898-915.

[14] D. R. Farkas, Poisson polynomial identities, Comm. Algebra, 26(2), 1998, 401-416.

[15] D. R. Farkas, Poisson polynomial identities II, Arch. Math., 72(4), 1999, 252-260.

[16] B. Fresse, Théorie des opérades de Koszul et homologie des algèbres de Poisson, Ann. Math. Blaise Pascal, 13(2), 2006, 237-312.

[17] A. Giambruno, V. M. Petrogradsky, Poisson identities of enveloping algebras, Arch. Math., 87(6), 2006, 505-515.

[18] V. Ginzburg, D. Kaledin, Poisson deformations of symplectic quotient singularities, Adv. Math., 186(1), 2004, 1-57.

[19] M. Goze, E. Remm, Poisson algebras in terms of non-associative algebras, J. Algebra, 320(1), 2008, 294-317. 
[20] J. Huebschmann, Poisson cohomology and quantization, J. Reine Angew. Math., 408, 1990, 57-113.

[21] M. Kontsevich, Deformation quantization of Poisson manifolds, Lett. Math. Phys., 66(3), 2003, 157-216.

[22] L. A. Kurdachenko, J. Otal, A. A. Pypka, Relationships between factors of canonical central series of Leibniz algebras, Eur. J. Math., 2(2), 2016, 565-577.

[23] L. A. Kurdachenko, J. Otal, I. Ya. Subbotin, On some properties of the upper central series in Leibniz algebras, Comment. Math. Univ. Carolin., 60(2), 2019, 161-175.

[24] L. A. Kurdachenko, A. A. Pypka, I. Ya. Subbotin, On some relations between the factors of the upper and lower central series in Lie algebras, Serdica Math. J., 60(2-3), 2015, 293-306.

[25] L. A. Kurdachenko, I. Ya. Subbotin, On the relationships between the factors of upper and lower central series in groups and other algebraic structures, Note Mat., 36(1), 2016, 35-50.

[26] L.-C. Li, Classical r-matrices and compatible Poisson structures for Lax equations on Poisson algebras, Comm. Math. Phys., 203(3), 1999, 573-592.

[27] A. Lichnerowicz, Les variétés de Poisson et leurs algèbres de Lie associées, J. Differential Geom., 12(2), 1977, 253-300.

[28] L. Makar-Limanov, I. Shestakov, Polynomial and Poisson dependence in free Poisson algebras and free Poisson fields, J. Algebra, 349(1), 2012, 372-379.

[29] L. Makar-Limanov, U. Turusbekova, U. Umirbaev, Automorphisms and derivations of free Poisson algebras in two variables, J. Algebra, 322(9), 2009, 3318-3330.

[30] L. Makar-Limanov, U. Umirbaev, Centralizers in free Poisson algebras, Proc. Amer. Math. Soc., 135(7), 2007, 1969-1975.

[31] L. Makar-Limanov, U. Umirbaev, The Freiheitssatz for Poisson algebras, J. Algebra, 328(1), 2011, 495-503.

[32] M. Markl, E. Remm, Algebras with one operation including Poisson and other Lie-admissible algebras, J. Algebra, 229(1), 2006, 171-189.

[33] S. P. Mishchenko, V. M. Petrogradsky, A. Regev, Poisson PI algebras, Trans. Amer. Math. Soc., 359(10), 2007, 4669-4694.

[34] I. Z. Monteiro Alves, V. M. Petrogradsky, Lie structure of truncated symmetric Poisson algebras, J. Algebra, 488, 2017, 244-281.

[35] B. H. Neumann, Groups with finite classes of conjugate elements, Proc. Lond. Math. Soc., 3(1), 1951, 178-187.

[36] A. Odzijewicz, Hamiltonian and quantum mechanics, Geom. Topol. Monogr., 17, 2011, 385-472.

[37] A. I. Ooms, The Poisson center and polynomial, maximal Poisson commutative subalgebras, especially for nilpotent Lie algebras of dimension at most seven, J. Algebra, 365, 2012, 83-113.

[38] A. Polishchuk, Algebraic geometry of Poisson brackets, J. Math. Sci., 84(5), 1997, 1413-1444.

[39] R. T. Prosser, Poisson brackets and commutator brackets. I, Proc. Amer. Math. Soc., 62(2), 1977, 305-309. 
[40] R. T. Prosser, Poisson brackets and commutator brackets. II, Proc. Amer. Math. Soc., 62(2), 1977, 310-315.

[41] S. M. Ratseev, Growth in Poisson algebras, Algebra Logic, 50(1), 2011, 46-61.

[42] S. M. Ratseev, Poisson algebras of polynomial growth, Sib. Math. J., 54(3), 2013, 555-565.

[43] S. M. Ratseev, Correlation of Poisson algebras and Lie algebras in the language of identities, Math. Notes, 96(3-4), 2014, 538-547.

[44] S. M. Ratseev, On minimal Poisson algebras, Russian Math., 59(11), 2015, 54-61.

[45] I. P. Shestakov, Quantization of Poisson superalgebras and speciality of Jordan Poisson superalgebras, Algebra Logic, 32(5), 1993, 309-317.

[46] S. Siciliano, Solvable symmetric Poisson algebras and their derived lengths, J. Algebra, 543, 2020, 98-110.

[47] U. Umirbaev, Universal enveloping algebras and universal derivations of Poisson algebras, J. Algebra, 354(1), 2012, 77-94.

[48] I. Vaisman, Lectures on the geometry of Poisson manifolds, Progress in Mathematics, 118, Birkhäuser, Basel and Boston, 1994.

[49] M. Van den Bergh, Double Poisson algebras, Trans. Amer. Math. Soc., 360(11), 2008, 5711-5769.

[50] M. R. Vaughan-Lee, Metabelian BFC p-groups, J. Lond. Math. Soc., 5(4), 1972, 673-680.

[51] M. Vergne, La structure de Poisson sur l'algèbre symétrique d'une algèbre de Lie nilpotente, Bull. Soc. Math. France, 100, 1972, 301-335.

[52] A. Weinstein, Lecture on Symplectic Manifolds, CBMS Regional Conference Series in Mathematics 29, Amer. Math. Soc., Providence, R.I., 1979.

\section{CONTACT INFORMATION}

L. A. Kurdachenko, Oles Honchar Dnipro National University,

A. A. Pypka

Gagarin ave., 72, Dnipro, 49010, Ukraine

E-Mail(s): Ikurdachenko@i.ua, sasha.pypka@gmail.com

\section{Ya. Subbotin}

National University, 5245 Pacific Concourse

Drive, Los Angeles, CA 90045-6904, USA

$E$-Mail(s): isubboti@nu.edu

Received by the editors: 15.01.2021. 